\title{
A STUDY OF MITOCHONDRIAL MEMBRANES IN RELATION TO ELEMENTARY PARTICLES
}

\author{
S. K. MALHOTRA AND R. T. EAKIN \\ Division of Biology, California Institute of Technology, Pasadena, California, U.S.A.
}

\section{SUMMARY}

Elementary particles that commonly have been seen by electron microscopy to be attached by stalks to mitocbondrial cristae in negatively stained preparations, were not apparent in similarly stained mitochondria from exponentially growing wild-type Neurospora crassa when these were isolated in sucrose solution containing $\mathrm{I} \times \mathrm{IO}^{-3} \mathrm{M}$ EDTA. However, elementary particles were easily demonstrable in electron micrographs if the mitochondria were isolated without EDTA in the sucrose solution. A biochemical study indicated that both kinds of mitochondrial preparations, isolated in the presence or absence of EDTA, had about the same capacity for oxidative phosphorylation. Observations on rat-liver mitochondria also suggested that the stalked elementary particles were more easily demonstrated if the preparation was made in the absence of EDTA. It was difficult to demonstrate elementary particles in wild-type Neurospora mitochondria isolated with or without EDTA and subsequently prepared for electron microscopy by spreading on the surface of an aqueous solution of potassium phosphotungstate. Elementary particles could be demonstrated in poky Neurospora mitochondria isolated with EDTA if the mitochondria were spread on the surface of an aqueous solution of phosphotungstate. It was concluded that biochemical functions associated with elementary particles are independent of structural configuration as seen by electron microscopy.

\section{INTRODUCTION}

The presence of elementary particles of constant dimensions (approximately 80$100 \AA$ in diameter) is a regular feature of isolated and negatively stained mitochondria of animal and plant cells. These elementary particles are seen in electron micrographs attached to the membranes by a stalk approximately $35 \AA$ wide and $40 \AA$ long and protruding towards the matrix of the mitochondrion (see Stasny \& Crane, I964; Parsons, 1965; Green \& Perdue, 1966a,b). During an investigation on isolated mitochondrial fractions (henceforth referred to as mitochondria) from Neurospora crassa stained negatively with phosphotungstate, it was observed that the stalked elementary particles were not apparent in electron micrographs, though these particles had been observed earlier by Stoeckenius (1963) in similarly stained mitochondria isolated from the same organism. However, the sucrose medium used in the procedure for isolation of mitochondria was slightly different from that used by Stoeckenius, in that $\mathrm{I} \times \mathrm{IO}^{-3} \mathrm{M}$ EDTA (ethylenediaminetetra-acetate) was present. When EDTA was omitted from our sucrose medium, the stalked elementary particles were demonstrable in electron micrographs of Neurospora mitochondria. 
EDTA is commonly used in media for isolation of mitochondria because its capacity to chelate divalent cations, particularly $\mathrm{Ca}^{2+}$ and $\mathrm{Mg}^{2+}$, prevents swelling (Lynn, Fortney \& Brown, I964; Parsons, 1965); and mitochondria isolated in such media exhibit their normal biochemical activity. The elementary particles have been demonstrated to be associated with the presence of enzymes of oxidative phosphorylation and mitochondrial ATPase (Racker, Chance \& Parsons, 1964; also see Parsons, 1965). It was therefore desirable to know whether the apparent presence or absence of the stalked elementary particles in electron micrographs of Neurospora mitochondria was reflected in biochemical functions. Thus mitochondria isolated with and without EDTA in the sucrose medium have been investigated biochemically and by electron microscopy.

\section{MATERIALS AND METHODS}

Mitochondria from exponentially growing mycelia of the wild-type strain $25 a$ of Neurospora crassa were used in this investigation. The mycelia, when harvested, had been grown for 22-24 $\mathrm{h}$ and yielded on an average $5 \mathrm{~g}$ of wet weight per litre of Vogel's growth medium (Vogel, I956).

In earlier experiments $0.44 \mathrm{M}$ sucrose containing either $\mathrm{I} \times 10^{-3} \mathrm{M}$ EDTA or no EDTA was used for isolation of mitochondria, according to the procedure reported by Stoeckenius ( 1963 ). In later experiments a $0.25 \mathrm{M}$ sucrose medium containing $0.15 \%$ BSA (bovine serum albumin), with or without EDTA, was used, since the use of BSA had been recommended for isolation of functionally intact mitochondria from Neurospora (Hall \& Greenawalt, 1964). Cells were disrupted by grinding in sucrose and sand, using $2.5 \mathrm{ml}$ of sucrose and $0.5 \mathrm{~g}$ of sand for each gram of wet weight of mycelium. The cell walls, nuclei and sand were removed from the homogenate by centrifuging twice at $1000 \mathrm{~g}$ for $10 \mathrm{~min}$. Then a mitochondrial pellet was obtained by centrifugation at $15000 \mathrm{~g}$ for $30 \mathrm{~min}$. The pellet thus obtained was either washed with the sucrose medium, centrifuged and resuspended in a small volume of sucrose medium, or it was further purified in a linear sucrose density gradient as described by Luck ( 1963 ). The concentrations of BSA and EDTA (when added) in all the solutions used throughout the isolation procedure and in the subsequent biochemical experiments were maintained at $0.15 \%$ and $\mathrm{I} \times 10^{-3} \mathrm{M}$, respectively. The mitochondria were isolated and stored at about $4{ }^{\circ} \mathrm{C}$.

Some observations have also been made on mitochondria from the 'poky' $3627-2 a$ (mi-1) mutant of Neurospora, and from rat liver isolated with or without EDTA by using the procedure outlined above. These have been used for comparison with the mitochondria from the wild-type Neurospora. The poky mutant contains a cytoplasmically inherited amino-acid substitution in the mitochondrial structural protein which drastically affects the regulation of cytochrome synthesis (Woodward \& Munkres, I966). In young cultures there is a I6-fold excess of cytochrome $c$ accumulated and no detectable cytochrome $b$ or cytochrome $a$. Until these cytochromes begin to be synthesized, approximately $40 \mathrm{~h}$ after inoculation, respiration and oxidative phosphorylation are dependent on an alternative, non-cytochrome electron-transport chain 
(Tissieres, Mitchell \& Haskins, I952). Also, the regulation of lipid and phospholipid synthesis is defective as young cultures accumulate large quantities of free, unesterified fatty acids (Hardesty \& Mitchell, I 963 ). The poky mutant used in this investigation had grown for $40 \mathrm{~h}$ when harvested, and yielded about $0.3 \mathrm{~g}$ of wet weight per litre of Vogel's growth medium.

\section{Biochemical assays}

Reduced versus oxidized cytochrome difference spectra of the mitochondrial preparations were obtained by the method of Williams (1964).

Oxidative phosphorylation was measured by using the reaction mixture recommended by Hall \& Greenawalt (1964) for mitochondria of Neurospora. The assay system contained $0.1 \mathrm{ml}$ of $0.25 \mathrm{M}$ sodium succinate or $0.1 \mathrm{ml}$ of water (control), $0.1 \mathrm{ml}$ of $20 \mathrm{mg} / \mathrm{ml}$ yeast hexokinase in $0.3 \mathrm{M}$ glucose and $2.7 \mathrm{ml}$ of the following base solution: $125 \mathrm{~mm}$ sucrose, Io mM $\mathrm{MgCl}_{2}, 0.4 \% \mathrm{BSA}$, I mM ADP, Iо $\mathrm{mm} \mathrm{KH}_{2} \mathrm{PO}_{\text {, }}$, I mM NAD, with or without $\mathrm{I} \times 10^{-3} \mathrm{M}$ EDTA. The $\mathrm{pH}$ was 6.9. Reactions were started by adding $0 . \mathrm{Iml}$ of mitochondrial suspension. Oxygen consumption was measured polarographically with a Yellow Springs Instrument Co. Biological Oxygen monitor. Reactions were stopped by adding $0.1 \mathrm{ml}$ of $20 \%$ sodium deoxycholate followed by $0.5 \mathrm{ml}$ of $10 \%$ trichloroacetic acid. After the precipitate was centrifuged out, glucose-6-phosphate was determined by measuring NADPH production in the presence of glucose-6-phosphate dehydrogenase (Hohorst, I963). The difference between the glucose- 6 -phosphate content of a reaction mixture and that of a control was used as the measure of oxidative phosphate esterification.

\section{Electron microscopy}

Two procedures were adopted for negatively staining mitochondrial preparations: either (I) a diluted mitochondrial suspension was placed on the grid and then the staining solution was applied to the grid, or (2) the mitochondrial suspension was spread on the surface of an aqueous solution and then picked up on the grid.

(I) A drop of the mitochondrial suspension was diluted with several (2-10) drops of distilled water cooled to about $4{ }^{\circ} \mathrm{C}$. A drop of this diluted suspension was placed on a grid coated with a thin film of Formvar or Parlodion and carbon. This was allowed to stay for about I-2 $\mathrm{min}$ at room temperature, and most of the fluid was then removed from the grid by bringing a piece of filter paper along the edge of the grid. Without further waiting, 2-4 drops of a solution of potassium phosphotungstate ( $1-4 \%$ PTA brought to $\mathrm{pH} 6 \cdot 8-7$ with $10 \% \mathrm{KOH}$ ) were applied to the surface of the grid. As much of the fluid as possible was immediately removed by touching the edge of the grid with a piece of filter paper. The grid was then allowed to dry for at least $5 \mathrm{~min}$ before it was examined in the electron microscope.

(2) A drop of the mitochondrial suspension was applied to the surface of a glass slide, whose lower end was immersed in distilled water or in a solution of potassium phosphotungstate $\left(0.5^{-2} \%\right.$ PTA brought to $\mathrm{pH} \mathrm{6.8-7)}$ contained in a Petri dish. The mitochondrial suspension formed a film which was picked up on a coated grid. (When the spreading of mitochondrial suspension was done in distilled water, the staining solution was applied to the film after it was picked up on the grid.) The grid was 
allowed to dry for a few minutes before it was examined in the electron microscope. A part of this work on spreading mitochondria was done in collaboration with $\mathrm{Dr} \mathrm{K}$. B. Easterbrook.

For preparation of ultra thin sections, a small part of the mitochondrial pellet was routinely fixed in $2.5 \%$ glutaraldehyde and postfixed in $2 \%$ osmium tetroxide (both the fixatives were in $O^{\circ}{ }_{5} \mathrm{M}$ phosphate buffer). After dehydration in ethanol, the pellet was embedded in Araldite.

Throughout this biochemical and electron-microscopical investigation, both kinds of mitochondrial preparation, i.e. with and without EDTA, were treated alike as far as possible, so that a strict comparison of the two preparations could be made.

\section{RESULTS AND DISCUSSION}

The following results, unless otherwise specified, pertain to the mitochondria of exponentially growing mycelia of wild-type $N$. crassa.

Originally, mitochondria were isolated from Neurospora that had been stored at $-25^{\circ} \mathrm{C}$. Such mitochondrial preparations were isolated in sucrose containing EDTA. An electron-microscopic study of these preparations after staining on the grids with phosphotungstate ( $\mathrm{pH}$ 6.8-7) revealed many well-preserved mitochondria with an intact outer membrane and membranous projections into the mitochondrial matrix; the latter have been identified as cristae (Fig. I). In suitably stained preparations the cristae showed indications of subdivision into globular particles (face-on view) in some regions (arrows in Fig. 2). There is a wide variation in the size of the globules, from about 50 to $100 \AA$. In these mitochondria there was no indication of the presence of stalked elementary particles attached to the cristae. However, elementary particles are difficult to demonstrate in negatively stained preparations of intact mitochondria (Smith, 1963; Stasny \& Crane, 1964; Parsons, 1965). But even in swollen and disrupted mitochondria, the stalked elementary particles were not apparent (Figs. 3, 7). Essentially similar results were obtained with mitochondria isolated immediately after harvesting the mycelia. No noticeable difference in the structure of mitochondria was observed if they were stored at $4{ }^{\circ} \mathrm{C}$ up to a week after they were isolated (compare with Sjöstrand, Andersson-Cedergren \& Karlsson, 1964).

Mitochondria prepared in the absence of EDTA, but otherwise under conditions comparable to those used for preparations with EDTA, were similar in appearance to those described by Stoeckenius (1963) and were mostly swollen and disrupted. Figs. 4 and 8 show that the elementary particles are clearly demonstrated as stalked bodies attached to the membranes. It is not intended to suggest that every mitochondrial membrane examined from these preparations has revealed stalked elementary particles, but they have been much more readily demonstrated in preparations without EDTA. As this difference in the two kinds of preparation has been observed in all of several experiments, it seems unlikely that there are two types of mitochondria, one which readily shows stalked elementary particles and the other that does not. Moreover, intact or well-preserved mitochondria isolated without EDTA had in general a much more granular appearance than those isolated with EDTA. When mitochondria isolated in medium without EDTA were incubated in the presence 
of $\mathrm{I} \times 1 \mathrm{IO}^{-3} \mathrm{M}$ EDTA at $4{ }^{\circ} \mathrm{C}$ for $\mathrm{I} 2 \mathrm{~h}$, the elementary particles were still demonstrable in electron micrographs (Fig. 5, the stalks are not particularly evident in this micrograph). This indicates that EDTA does not obviously damage or destroy the elementary particles in Neurospora mitochondria, so that their absence from the electron micrographs does not necessarily represent the loss of the material constituting the elementary particles. When mitochondria isolated in medium with or without EDTA were studied in sections after fixation in glutaraldehyde and osmium tetroxide (both in $0.15 \mathrm{M}$ phosphate buffer, $\mathrm{pH} 7 \cdot 2$ ), there were no obvious differences in the two kinds of preparation.

Table I. Oxidative phosphorylation by isolated mitochondria using succinate as a substrate

\begin{tabular}{cccc}
\hline Mitochondrial preparation & $\begin{array}{l}\text { Phosphate } \\
\text { esterification } \\
(\mu \mathrm{mole} / \mathrm{mg} / \mathrm{h})\end{array}$ & $\begin{array}{c}\text { Oxygen } \\
\text { consumption } \\
(\mu \mathrm{mole} / \mathrm{mg} / \mathrm{h})\end{array}$ & $\begin{array}{c}\text { P:O } \\
(\mu \text { atom P } / \mu \text { atom O) }\end{array}$ \\
\hline Without EDTA & 3.9 & 3.4 & 0.57 \\
With I $\times$ I $^{-3}$ M EDTA & 3.5 & 4.8 & 0.36 \\
Measurements were carried out in the presence of $8.3 \mathrm{~mm}$ succinate. \\
\hline
\end{tabular}

Table 2. Effect of additional succinate and externally added cytochrome $c$ on the rate of oxygen consumption

\begin{tabular}{|c|c|c|c|c|}
\hline $\begin{array}{l}\text { Mitochondrial } \\
\text { preparation }\end{array}$ & $\begin{array}{l}\text { Succinate } \\
\text { conc. (mM) }\end{array}$ & $\begin{array}{c}\text { Cytochrome } c \\
\text { conc. } \\
(\mathrm{mg} / 100 \mathrm{ml})\end{array}$ & $\begin{array}{l}\text { Mito- } \\
\text { chondrial } \\
\text { protein } \\
\text { (mg) }\end{array}$ & $\begin{array}{c}\text { Oxygen } \\
\text { consumption } \\
\text { ( } \mu \text { mole } / \\
\text { min })\end{array}$ \\
\hline \multirow[t]{3}{*}{ Without EDTA } & $8 \cdot 3$ & o & 2 & 0.086 \\
\hline & $16 \cdot 1$ & 0 & 2 & $0.09 \mathrm{I}$ \\
\hline & $15 \cdot 6$ & 0.03 & 2 & 0.127 \\
\hline \multirow[t]{3}{*}{ With $\mathrm{I} \times 1 \mathrm{I}^{-3} \mathrm{M}$ EDTA } & $8 \cdot 3$ & $\circ$ & 2 & O.IOI \\
\hline & $I 6 \cdot I$ & 0 & 2 & $0 \cdot 120$ \\
\hline & I 5.6 & 0.03 & 2 & 0.146 \\
\hline
\end{tabular}

In order to determine whether the presence or absence of elementary particles on stalks attached to the membranes of the cristae in electron micrographs was reflected in the functional state of the two preparations of mitochondria, a biochemical investigation was undertaken. For this purpose, mitochondria isolated and banded on a linear sucrose density gradient were used on the same day that they were prepared. Cytochrome difference spectra of mitochondria prepared with or without EDTA were the same, and therefore the two mitochondrial preparations had essentially the same cytochrome composition.

Both preparations of mitochondria, with and without EDTA, were functional, as they had the capacity for oxidative phosphorylation, indicated in Table I. Mitochondria prepared in the presence of EDTA exhibited a slightly lower $P: O$ ratio, 
but the phosphorylation rate was about the same as that obtained with mitochondria isolated without EDTA. Thus the presence of EDTA in the isolation medium seemed to have little effect on the rate of phosphate esterification but enhanced the rate of oxygen consumption by the mitochondria, thereby reducing the $P: O$ ratio observed in mitochondria isolated with EDTA. To determine if the difference in the rate of oxygen consumption in the two kinds of preparation was due to differences in the intramitochondrial concentration of substrate or cytochrome $c$, the rate of oxygen consumption was measured after increasing the substrate concentration and after adding horse heart cytochrome $c$. The difference in the rates was not altered, as shown in Table 2. Additional succinate and cytochrome $c$ slightly stimulated the rate of oxygen consumption, but both types of preparation responded similarly, thus leaving the difference originally observed unaltered. The experimental conditions were kept constant, apart from the presence or absence of EDTA; and as the two kinds of preparation of mitochondria were obtained from mycelia that had been cultured and harvested from the same carboy, the above data are indicative of the effect of EDTA on mitochondrial function. As mitochondria isolated with or without EDTA have about the same capacity for oxidative phosphorylation, EDTA at the concentration used in this investigation does not cause obvious disorganization of their biochemical properties. It is not clear from the present study how the presence of EDTA in the sucrose medium affects the structure of the mitochondrial membranes and consequently the appearance of the stalked elementary particles in the electron micrographs of negatively stained mitochondria. It is conceivable that removal of $\mathrm{Ca}^{2+}$ and $\mathrm{Mg}^{2+}$ by EDTA may cause shrinkage of mitochondria in Neurospora and the elementary particles are, or become in this state, part of the membrane and as such do not protrude into the matrix. This effect of EDTA may not be restricted only to the mitochondria of exponentially growing mycelia of Neurospora. In experiments with rat liver, the stalked elementary particles were generally demonstrated more clearly in electron micrographs of mitochondria isolated without (Fig. 10) than with (Fig. 9) EDTA in the sucrose medium. Moreover, Sjöstrand et al. (1964) had reported that the stalked elementary particles were very infrequently found in mitochondria isolated in EDTAcontaining medium from the rat heart and kidney.

Preliminary observations suggested that mitochondria isolated from mycelia that were not growing in exponential phase did not show such a noticeable effect of EDTA on their structure as demonstrated by negative staining. The reason for this difference in structure between mitochondria isolated from the non-exponential phase of growth and from the exponential phase of growth is not apparent from the results of present investigation. It is conceivable that there may be differences in the permeability properties of mitochondria in the two phases of growth of Neurospora, which impart different spreading properties to the mitochondria of these phases. Mitochondria from the non-exponential phase isolated in sucrose containing EDTA and stained with phosphotungstate probably swell more than the mitochondria similarly prepared from the exponential phase. This difference in the degree of swelling may affect the morphological state of the elementary particles to the extent that they could be demonstrated by electron microscopy in one phase but not in the other. 
Mitochondria isolated from exponentially growing mycelia and spread on the surface of an aqueous solution (with or without phosphotungstate, see Materials and Methods) show structural features different from mitochondria stained directly on the grid. Neither type of mitochondrial preparation, that is, with or without EDTA, would spread easily as compared with mitochondria from, for example, liver (see Parsons, 1965). Both kinds of mitochondrial preparation spread and stained in this way looked very similar to those illustrated in Figs. I and 2 or like Fig. 7, and the stalked elementary particles were not shown. Apparently the poor spreading left the mitochondria in a state such that the stalked elementary particles were not demonstrable. The difficulty in demonstrating the stalked elementary particles in mitochondria isolated with EDTA and stained by the first described method-that is, directly on the grids without spreading on the surface of an aqueous solution-also may have been a result of high density (tight packing) of the contents of the mitochondrial matrix and membranous material as a consequence of poor spreading.

Mitochondria isolated from the poky Neurospora mutant spread out easily on the surface of aqueous solution (observations made in collaboration with K. B. Easterbrook), perhaps as a result of the differences in biochemical composition as compared to wild-type (see Materials and Methods). When spread on the surface of an aqueous solution and stained with phosphotungstate, poky mitochondria isolated with EDTA clearly showed the stalked elementary particles (Fig. 6); but if these mitochondria were stained on the grids, no stalked elementary particles were observed. Judging from their distribution in electron micrographs, the stalked elementary particles seem to be fewer in mitochondria from the poky mutant (Fig. 6) than in mitochondria from the wild-type Neurospora (Figs. 4, 8) or from rat liver (Fig. I0).

The conclusion from this work is that the presence of EDTA during isolation directly or indirectly influences the physical state of the elementary particles in mitochondria, but that it does not greatly alter the capacity for oxidative phosphorylation. Furthermore the presence of stalked elementary particles may be dependent upon the physiological state of the mitochondria and the procedure of negative staining. Thus the apparent presence or absence of stalked elementary particles in electron micrographs may not in itself have a physiological significance.

We are grateful to $\mathrm{Dr} H$. K. Mitchell for his valuable comments and advice in writing this manuscript. This work has been supported by grants from the U.S. Public Health Service (GM 06965), the National Science Foundation (GB 2055), and a U.S. Public Health Service Predoctoral Fellowship to R.T.E. (GM I9913).

\section{REFERENCES}

Green, D. E. \& Perdue, J. F. (1966a). Membranes as expressions of repeating units. Proc. natn. Acad. Sci. U.S.A. 55, 1295-1 302.

Green, D. E. \& Perdue, J. F. (I966b). Correlation of mitochondrial structure and function. Ann. N.Y. Acad. Sci. 137, 667-684.

Hall, D. O. \& Greenawalt, J. W. (1964). Oxidative phosphorylation by isolated mitochondria of Neurospora crassa. Biochem. biophys. Res. Commun. 17, 565-569.

Hardesty, B. A. \& Mitchell, H. K. ( 1963$)$. The accumulation of free fatty acids in poky, a maternally inherited mutant of Neurospora crassa. Archs Biochem. Biophys. 100, 330-334. 
HoHorST, H. (1963). D-Glucose-6-phosphate and D-fructose-6-phosphate. Determination with glucose-6-phosphate dehydrogenase and phosphoglucose isomerase. In Methods of Enzymatic Analysis (ed. H. U. Bergmeyer), pp. 134-1 38. New York: Academic Press.

Luck, D. J. L. (1963). Genesis of mitochondria in Neurospora crassa. Proc. natn. Acad. Sci. U.S.A. 49, 233-240.

LYNN, W. S., ForTNeY, S. \& Brown, R. S. (1964). Osmotic and metabolic alterations of mitochondrial size. F. Cell Biol. 23, I-8.

Parsons, D. F. (1965). Recent advances correlating structure and function in mitochondria. Int. Rev. exp. Path. 4, I-54.

Racker, E., Chance, B. \& Parsons, D. F. (1964). Correlation of structure and function of submitochondrial units in oxidative phosphorylation. Fedn Proc. Fedn Am. Socs exp. Biol. 23, 431 ,

SjUstrand, F. S., Andersson-Cedergren, E. \& Karlsson, U. (ig64). Myelin-like figures formed from mitochondrial material. Nature, Lond. 202, 1075-1078.

SмIтH, D.S. (1963). The structure of flight muscle sarcosomes in the blowfly Calliphora erythrocephala (Diptera). F. Cell Biol. 19, I $15^{-1} 3^{8}$.

StASNY, J. T. \& Crane, F. L. (I963). The effect of sonic oscillation on the structure and function of beef heart mitochondria. I. Cell Biol. 22, 49-62.

Stozckenius, W. (1963). Some observations on negatively stained mitochondria. f. Cell Biol. I7, 443-454.

Tissieres, A., Mitchell, H. K. \& Haskins, F. A. (1953). Studies on the respiratory system of the poky strain of Neurospora. $\mathcal{J}$. biol. Chem. 205, 423-433.

Vogel, H. J. (1956). A convenient growth medium for Neurospora. Microb. Genet. Bull. No. r3, pp. $42-43$.

WiLLIAMS, J. N. (I964). A method for the simultaneous quantitative estimation of cytochromes $a, b, c_{1}$ and $c$ in mitochondria. Archs Biochem. Biophys. 107, 537-543.

Woodward, D. O. \& Munkres, K. D. (Ig66). Alterations of a maternally inherited mitochondrial structural protein in respiratory-deficient strains of Neturospora. Proc. natn. Acad. Sci. U.S.A. 55, 872-88o.

(Received I I October I966)

All the electron micrographs included in this article are of mitochondrial fractions stained with potassium phosphotungstate $(\mathrm{pH} 6 \cdot 8-7)$ on the Formvar or Parlodion and carbon-coated grids, except Fig. 6 which shows a mitochondrial fraction spread on the surface of distilled water and then picked up on the grid and stained in phosphotungstate $\left(\mathrm{pH}_{7}\right)$.

Fig. I. From a mitochondrial fraction isolated in sucrose with EDTA, showing typical appearance of a fairly well-preserved mitochondrion. Double arrows indicate the outer limiting membrane. In the right upper corner part of a disrupted mitochondrion is seen. Note the absence of stalked elementary particles. $\times 220000$.

Fig. 2. Part of a well-preserved mitochondrion from a preparation similar to that in Fig. I. Arrows indicate possible globular particles in or on the membranes of the cristae. The outer mitochondrial membrane is seen running across the entire width near the upper limit of this micrograph. $\times 285000$.

Fig. 3. From a mitochondrial fraction isolated in sucrose with EDTA. Note that the membranes do not show any stalked elementary particles attached to them, even though the mitochondria are disrupted. $\times 165000$. 
fournal of Cell Science, Vol. 2 , No. 2
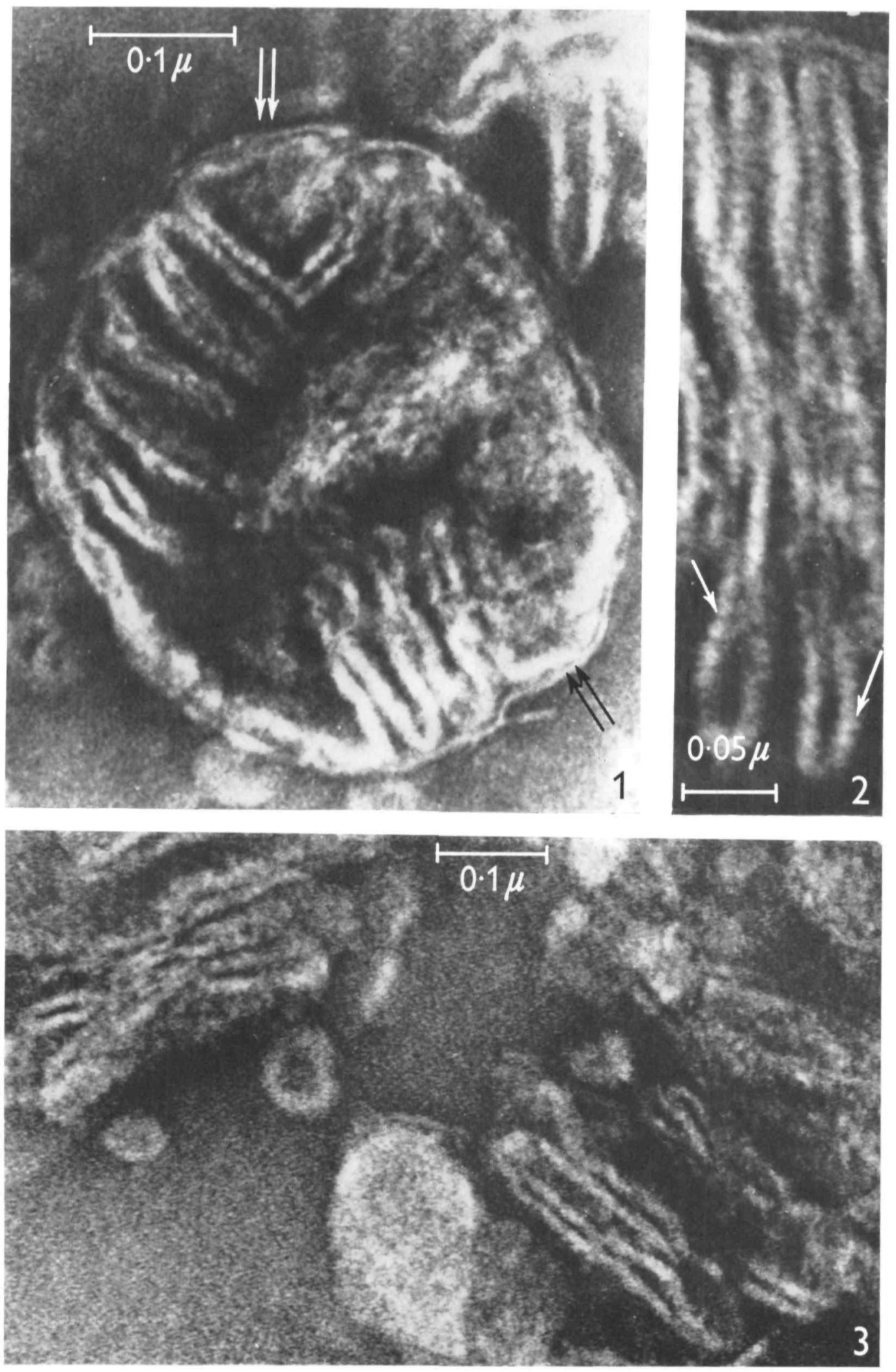

S. K. MALHOTRA AND R. T. EAKIN

(Facing p. 212) 
Fig. 4. From a mitochondrial fraction isolated in sucrose without ED'TA. Note the rows of elementary particles (arrows) along the sides of negatively stained ribbon-like membranes. $\times 216000$.

Fig. 5. From a mitochondrial fraction isolated in sucrose without EDTA and then incubated in EDTA for $12 \mathrm{~h}$ at $4{ }^{\circ} \mathrm{C}$. Stalked elementary particles are indicated by an arrow. $\times 268000$.

Fig. 6. From a mitochondrial fraction of the poky mutant isolated in $0.44 \mathrm{M}$ sucrose with EDTA and spread on the surface of an aqueous solution. Note that the mitochondria are very well spread out and broken; and elementary particles are seen in close proximity to the membranes (arrows) but their attachment to the membranes is not clear. $\times 335000$ 
Fournal of Cell Science, Vol. 2, No. 2
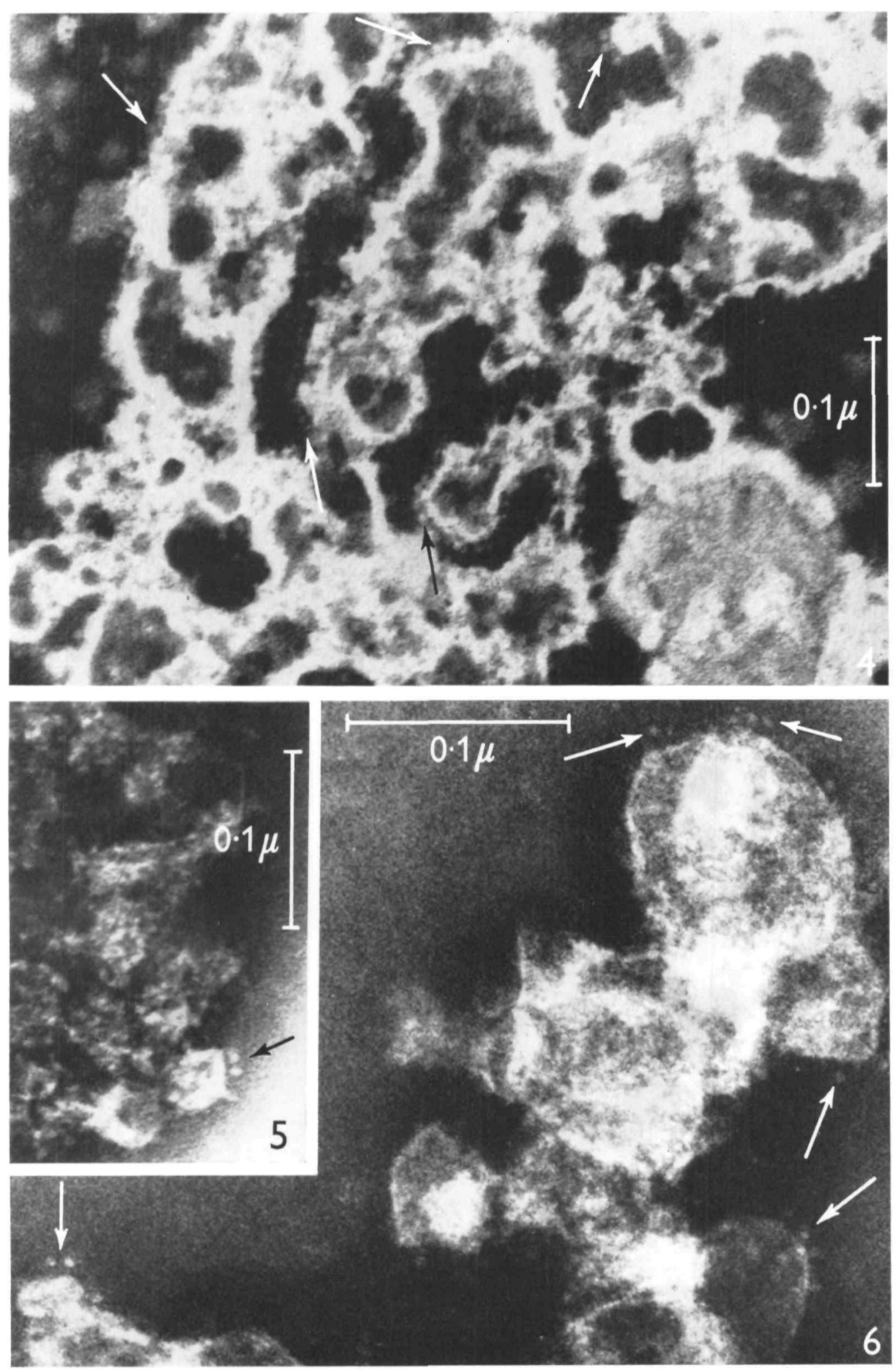

S. K. MALHOTRA AND R. T. EAKIN 
Figs. 7, 8. From mitochondrial fractions isolated from mycelia harvested from the same carboy. $\times 216000$

Fig. 7. With EDTA. The contents of the mitochondrion are dense and the structural details are not easy to interpret.

Fig. 8. Without EDTA. The contents of the mitochondrion are dispersed and the elementary particles are shown attached to the sides of the membranes (arrows).

Figs. 9, ro. From mitochondrial fractions of rat liver. $\times 268000$.

Fig. 9. Isolated in $0.44 \mathrm{M}$ sucrose medium with EDTA. The arrows indicate flattened membranes having granular appearance but no distinct stalked elementary particles are present.

Fig. Io. Isolated in $0.44 \mathrm{M}$ sucrose medium without ED'TA. Note that the stalked elementary particles (arrows) are clearly shown. 
Fournal of Cell Science, Vol. 2, No. 2
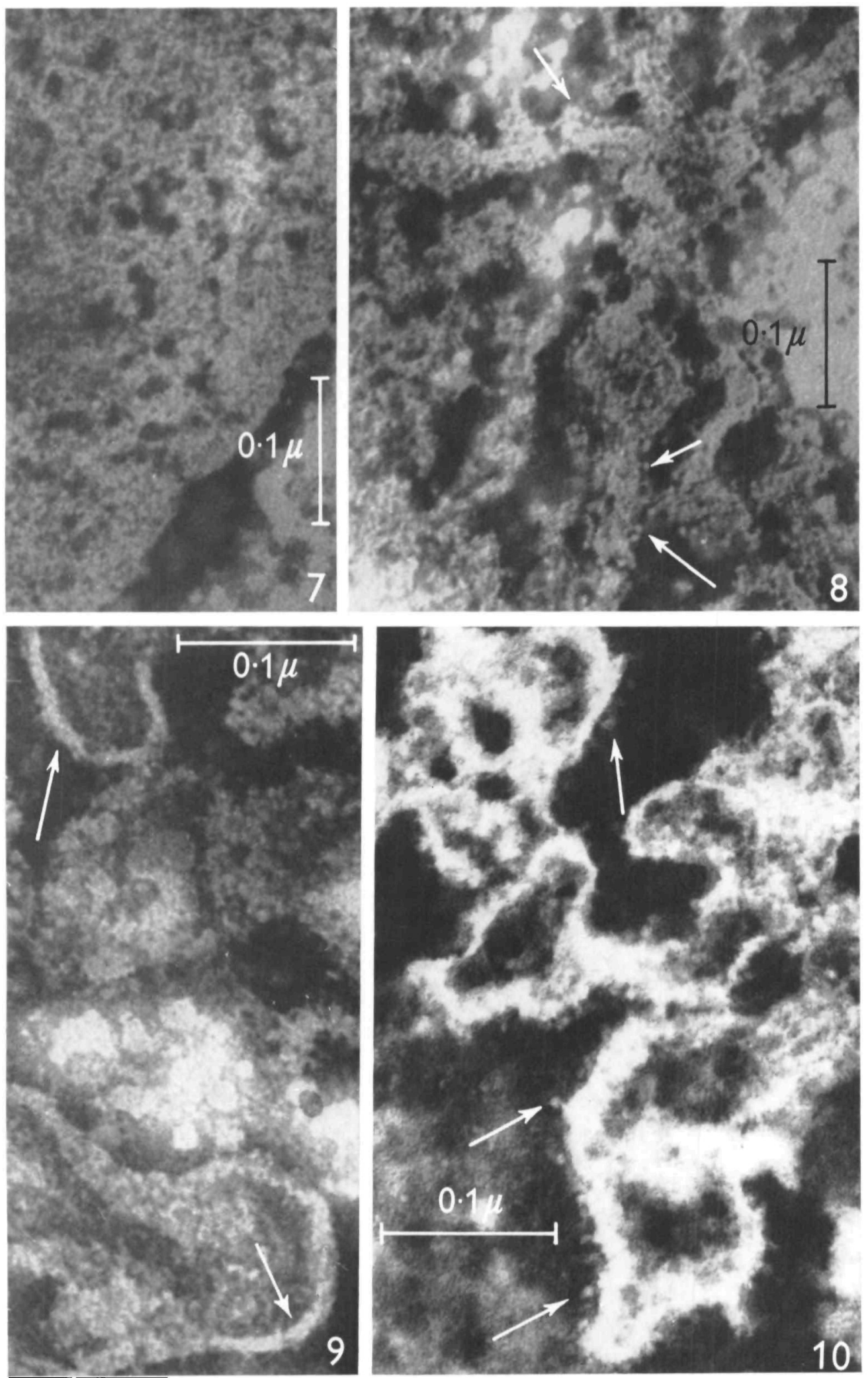

S. K. MALHOTRA AND R. T. EAKIN 
